CLINICAL ALERT

\title{
Nososcomial transmission of viral haemorrhagic fever in South Africa
}

\begin{abstract}
G A Richards
Prof. Guy Richards is currently Academic Head and Professor of the Division of Critical Care in the Faculty of Health Sciences, University of the Witwatersrand, Johannesburg, South Africa. He is Director of the Department of Critical Care at Charlotte Maxeke Johannesburg Academic Hospital, Johannesburg, and a chief physician in the Department of Medicine and Pulmonology there. Prof. Richards specialised in internal medicine in 1985 and subsequently qualified as a pulmonologist and intensivist, and was awarded his PhD in medicine in 1992 from the University of the Witwatersrand.
\end{abstract}

Corresponding author: G A Richards (guy.richards@wits.ac.za)

Recent events in West Africa have highlighted the potential for the viral haemorrhagic fevers (VHFs) to cause considerable mortality and morbidity among heathcare workers. However, this is not a new threat as, although the risk is currently increased, it has always been present. In South Africa (SA) the only endemic haemorrhagic fever is Crimean-Congo haemorrhagic fever, transmitted by the Hyalomma tick, which is ubiquitous in cattle farming areas. Johannesburg, the commercial and transport hub of SA, is unusual in that all cases of VHF seen there are imported, either from rural areas in SA or from countries to the north. Johannesburg functions as the gateway to and from the rest of Africa, and as a destination for more affluent residents of neighbouring countries seeking medical attention. Numerous outbreaks of nosocomial infection have occurred in SA, and these are described in the form of brief case reports.

S Afr Med J 2015;105(9):709-712. DOI:10.7196/SAMJnew.8168

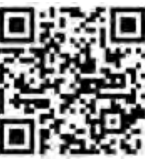

Emergency department personnel must be constantly aware of the risk of exposure to a patient with a communicable viral haemorrhagic fever (VHF). This requires that they are familiar with the symptoms and signs of this group of formidable diseases and that they should also be aware of geographical regions where VHFs are endemic or, as has lately been the case, epidemic. The subsequent care of a patient with a suspected or proven VHF requires strict attention to infection control and access to appropriate personal protective equipment (PPE). The following case reports illustrate the possibility of exposure in South Africa (SA) and highlight the potential for loss of life if these diseases are not recognised or infection control is inadequate.

\section{Case reports}

\section{Marburg virus, 1975}

The first documented case of Marburg virus in Africa, and the first since the original epidemic in West Germany and Yugoslavia in 1967, occurred in Johannesburg in February 1975. ${ }^{[1,2]}$ The primary case, a young Australian, was admitted to Johannesburg Hospital $(\mathrm{JH})$ after having toured what was then Rhodesia (now Zimbabwe). He had a high fever, myalgia, vomiting, diarrhoea, a maculopapular rash, hepatitis, leucopenia and thrombocytopenia, and died on the 7 th day after presentation without a diagnosis, from disseminated intravascular coagulation (DIC)-related haemorrhage and hepatic failure. Because a VHF was suspected and appropriate infection control practices had not initially been observed, all 35 direct contacts were isolated in the Johannesburg Fever Hospital (JFH), and all less close contacts kept under daily surveillance.

The travelling companion of the first patient subsequently became ill with similar symptoms, and was also admitted to JFH. She recovered after 10 days following a stormy course, having been cared for by volunteers from among the staff who had been placed in isolation. Eight days later, a 20-year-old nurse who had cared for the first patient before initiation of appropriate barrier nursing, and had in addition handled wet facial tissues while consoling patient 2 on the death of her travelling companion, also became ill. She continued to work at JFH, only stopping 24 hours later when symptoms became intolerable. Over the next 2 days she developed diarrhoea, fever, backache, myalgia, a rash and hepatitis. By day 7 she fortunately began to improve and was later discharged. The travelling companion could have contracted the disease prior to admission to hospital, and the nurse, although having had unprotected contact, had no more contact than many others.

\section{Crimean-Congo haemorrhagic fever}

On 28 August 1984, a 26-year-old man presented to a hospital near Cape Town, with haemorrhagic manifestations. ${ }^{[3]} \mathrm{He}$ was subsequently transferred to an intensive care unit (ICU) in an academic hospital, where he died from multiple organ dysfunction on 4 September. The diagnosis was confirmed by isolation of the Crimean-Congo haemorrhagic fever (CCHF) virus from admission bloods, and from liver tissue removed post mortem. This was prior to the availability of identification techniques utilising the polymerase chain reaction (PCR), and initially no antibodies were detected. Seven healthcare workers (HCWs) subsequently became ill with CCHF, primarily because stringent isolation procedures were instituted only 36 hours after admission of the index patient, and initially numerous blood specimens were handled in the laboratory without precautions. The first three were nurses who had cared for the index patient during his first few hours in the ICU; two other nurses came into contact with contaminated material during the setting up of isolation procedures; a 37-year-old surgeon without known direct contact with the index patient but who had visited the ICU before isolation fell ill following an incubation period of 5 days and died 8 days later (the virus was subsequently isolated from his blood); and a nurse had a needlestick injury after initiation of contact precautions. ${ }^{[4]}$ Once appropriate infection control with isolation had been introduced, the latter was the only infection that occurred. 
In December 1985, a 47-year-old nurse at Kimberley Hospital died of CCHF. She had been caring for a 52-year-old man from the Kimberley area who had contracted CCHF by tick bite. This patient survived. There are few further details available about this incident (personal communication, Jacqueline Weyers, National Institute of Communicable Diseases, SA).

On 4 April 2006, a 24-year-old employed in a private pathology laboratory was admitted to the JH ICU with a 2-day history of an influenza-like illness (unpublished data, GAR). The only clinical abnormality was a platelet count of $7 \times 10^{9} / \mathrm{L}$. He had no travel history, no tick bites and no contact with animals. However, the laboratory in which he worked had processed specimens from an importer of birds and monkeys from the Democratic Republic of the Congo who had died on 1 April from an unidentified haemorrhagic illness. This patient had also been fishing in KwaZulu-Natal Province on 15 March, where he had been bitten by ticks; however, the PCR had been negative for VHFs. The laboratory worker had been on duty from 28 to 31 April. It was subsequently established that he had sorted through and repacked other specimens into the same refrigerator in which those from the first patient were stored (as had others in the laboratory), but there was no record of any specific exposure. He was tested for VHFs, all of which were negative, as were tests for other infectious diseases. However, barrier nursing procedures were maintained throughout. He was unresponsive to any form of therapy, and 3 days after admission he was intubated and mechanically ventilated after developing a large spontaneous hemopneumothorax. Twelve days after admission, repeat PCR for CCHF tested positive. Treatment with ribavirin was commenced when he developed a refractory hyperpyrexia of $41^{\circ} \mathrm{C}$, but he died shortly thereafter.

PCR tests are not always reliable initially, and VHFs should be considered in any HCW, including laboratory workers presenting with haemorrhagic manifestations. If an alternative diagnosis is not established, barrier precautions should be continued until a VHF is excluded. In this instance, no further transmission to staff members at JH occurred.

\section{Ebola Gabon virus}

In 1996 - 1997 an outbreak of Ebola virus disease (EVD) occurred in the Booué area of Gabon, with many patients subsequently transported to the capital, Libreville. ${ }^{[5]}$ This distant incident later impacted on SA. ${ }^{[6]}$ On 2 November 1996, a 46-year-old nursing sister/anaesthetic assistant became ill with fever and headache, requiring admission to a Johannesburg clinic on the 6th. She had no history of travel or insect bites, and as far as she was aware had not treated anyone with a communicable disease. The diagnosis was unclear, but because there was leucopenia $\left(2.4 \times 10^{9} / \mathrm{L}\right.$ on admission, decreasing to $0.8 \times 10^{9} / \mathrm{L}$ by 9 November) she was treated as having bacteraemia. She subsequently developed hepatitis and severe diarrhoea, and both the platelet count and renal function declined precipitously. Prior to definitive diagnosis, she developed haematemesis and melaena requiring active resuscitation, gastroscopy, intubation and mechanical ventilation. These were performed without precautions other than surgical masks, gloves, plastic aprons and in some cases permeable surgical gowns. When the bleeding continued, laparotomy was performed using standard surgical apparel, which confirmed an oedematous, haemorrhagic gastritis for which surgical intervention was not possible. The clinical features led to the suspicion of a VHF, and diagnostic investigations later confirmed the diagnosis of EVD by culture and immunofluorescence (again prior to the availability of PCR testing).

The patient was transferred to JH and the Department of Health was involved in logistical aspects. At $\mathrm{JH}$ she was found to have haematological evidence of DIC, and had visible petechiae and haematomas. She was continued on mechanical ventilation because of a low Glasgow Coma Score and because she became progressively more hypotensive. A Swan-Ganz catheter (SGC) was inserted; the initial pulmonary artery occlusion pressure was $22 \mathrm{mmHg}$, and both the left ventricular stroke work index and calculated systemic vascular resistance were low and remained so throughout the illness. The SGC did not measure continuous cardiac output, and these parameters were measured only occasionally to minimise exposure to bodily fluids. The platelet count remained low despite transfusions, methlyprednisone, broad-spectrum antibiotics, gammaglobulin and a relook laparotomy, and the temperature failed to settle. On day 23, she died from an intracranial haemorrhage. No antivirals or immune serum were utilised. Despite a strong antibody response and sustained improvement in liver enzymes, Ebola antigen was still detected in the blood up to day 20 and in the urine up to day 23 .

Once a definitive diagnosis had been made, a search was undertaken for the source of the infection. The only possible candidate was a doctor who had been brought to SA on a commercial flight from Libreville, with a history of 8 days of fever and diarrhoea. The nursing sister, although wearing gloves, surgical gown and mask, had been exposed to a great deal of blood from this patient during and after placement of a central venous catheter. However, there were no reports of needlestick injury and she had no more exposure than those who placed the catheter or performed the gastroscopy or laparotomy on the Gabonese doctor at a later stage.

The Gabonese doctor, who had been discharged, was found to have highly positive serology for Ebola, and antigen was also recovered from a semen sample. Although he denied knowledge of an Ebola outbreak in Gabon, it was determined that he had treated a patient from Booué, the epicentre of the epidemic. ${ }^{[5]}$

Little was known about Ebola at the time, and even experts in the field were uncertain as to the mode of transmission. It was presumed usually to be via contact with body fluids, but airborne transmission was considered possible, as breathable $0.8-1.0-2.0 \mu \mathrm{m}$ droplets had been documented to be highly infectious to rhesus monkeys exposed to the Reston and Zaire strains of Ebola virus in laboratory conditions. ${ }^{[7]}$ This was the first time JH staff had managed a patient with EVD, and initially there was considerable consternation. Highlevel barrier nursing was considered preferable and the patient was isolated, PPE was worn, and high-efficiency particulate air (HEPA)filtered respirators were used for invasive procedures. Case definitions were developed and distributed widely, as were definitions outlining what constituted significant exposure, and protocols were devised to optimise management.

\section{Novel arenavirus, 2008}

In 2008 an outbreak of an arenavirus infection similar to Lassa fever occurred. ${ }^{[8]}$ The primary case was a travel consultant in Zambia who became ill with fever and sore throat. Rapid deterioration required her transfer from Zambia to a Johannesburg clinic on 12 September, where she died 2 days later with cerebral oedema and multiple organ dysfunction. On 22 September, patient 2, an emergency medical therapist who had accompanied the primary case between Zambia and SA, became ill and on 27 September was transferred to the same clinic, where he died on 2 October. Given the similarity of clinical presentation, ${ }^{[9]}$ tests for VHF and other communicable diseases were performed, but were negative. Contact tracing was nevertheless initiated, which identified two further cases, a nursing sister and a cleaner, both of whom had been in contact with the primary patient. Both also tested negative for Lassa, CCHF, Marburg and Ebola viruses and had a rapid and fatal course. However, all four of these patients 
were subsequently found to be PCR-positive for a new arenavirus that was named the Lujo virus. On 5 October, another nursing sister who had been involved in the care of patient 2 became ill with fever, leucopenia, thrombocytopenia and a rash and tested positive for the arenavirus. ${ }^{[9]}$ This engendered considerable fear among all the staff, and in particular the nursing sister herself, who was a young mother. She was treated at the clinic at which she worked with assistance from the JH departments of Critical Care, Infectious Diseases and Infection Control. Oral ribavirin was initiated along with supportive therapy, but the hepatitis and thrombocytopenia worsened and she became confused. Consultations with international colleagues indicated that oral therapy was unlikely to achieve the minimum inhibitory concentrations necessary to eradicate the virus rapidly (personal communication, D G Bausch), so intravenous ribavirin was sourced from Valeant Pharmaceuticals (USA) in The Netherlands for the (iniquitous) price of USD45 000 for a 10-day course. ${ }^{[10]} \mathrm{On}$ initiation of this therapy there was prompt improvement, and the patient was subsequently discharged well.

It is not known how the two nursing sisters contracted the illness. All had used standard infection control procedures in the emergency department, including gown, gloves and surgical mask. It is possible that breaches occurred, because use of PPE is seldom sufficiently strictly supervised in the emergency room (ER), particularly prior to a definitive diagnosis being made. Neither reported needlestick injuries or contamination of open wounds with secretions. It is also not certain how the cleaner became ill; however, standard infection control procedures may not have been strictly followed, particularly if the cubicle was scrubbed down without use of gloves. This event emphasised the need for universal precautions for all patients admitted to the ER and ICU, regardless of the diagnosis.

\section{Discussion}

Many hospitals internationally are preparing for the management of patients with VHF, specifically because of the impact of the current Ebola outbreak. Many of these institutions have biosafety level 3 and 4 laboratories and designated isolation areas that enable them to deal with these formidable viruses safely. ${ }^{[1,12]}$

However, there is no telling where a VHF might appear, as most patients unfortunately do not know which are the specific referral hospitals, and do not know or suspect their own diagnoses. We therefore recommend that all hospitals have some awareness of the VHFs and have PPE available in the emergency department in order to obviate the dreadful consequences of what is, in many cases, the unnecessary loss of a colleague. We acknowledge that epidemics such as those in West Africa transiently increase adherence to infection control procedures. Nevertheless, even once the panic has died down, regular reinforcement of standard precautions should be practised in all hospitals to avoid a tendency to complacency ${ }^{[13]}$

\section{Reduction of risk}

A detailed travel history is mandatory for all patients presenting at any hospital, and if positive should include details of possible incubation periods and specific exposures. ${ }^{[14]}$ Hospitals in areas of greatest risk should have a case definition that alerts frontline doctors to the possibility of a VHF, and this should be modified according to each outbreak. While these diseases are rare, they must be recognised as possible in any HCW or laboratory worker presenting with acute onset of documented fever (oral $\geq 38^{\circ} \mathrm{C}$ or axillary $\geq 37.5^{\circ} \mathrm{C}$ ) and thrombocytopenia without any obvious alternative diagnosis, or in any patient with a history of travel to an area where these diseases are prevalent. Over 3 billion people travel by air annually, and as has recently been shown, any transmissible disease is but a few hours away. Whereas malaria is the most common diagnosis among travellers requiring hospitalisation for fever and thrombocytopenia, accounting for 27 - $42 \%$ of cases, this should not lead to a decrease in vigilance. ${ }^{[15,16]}$ Personal protection has become increasingly important because of the risk of exposure to transmissible diseases such as HIV, tuberculosis, influenza, anthrax and measles. ${ }^{[17-19]}$

\section{Preparation of hospitals}

Following the 1996 Ebola scare, JH was designated as a referral centre, and recently a number of other hospitals around the country have been similarly designated despite minimal instruction and training in infection control techniques. HCWs most likely to be exposed should receive regular training in the use of high-level PPE and in the reduction of environmental contamination. ${ }^{[20-22]} \mathrm{At} \mathrm{JH}$, now Charlotte Maxeke Johannesburg Academic Hospital (CMJAH), ICU and ER staff had been familiarised with disease recognition and PPE on a regular basis, even before the current Ebola outbreak. Infection control staff at CMJAH are also always available to instruct staff in the event of an outbreak, and in an ongoing fashion, between outbreaks.

An isolation area has been created capable of managing three critically ill patients in separate cubicles with a negative-pressure environment, with monitors no longer in use in the main ICU. This facility is maintained by infection control personnel and is permanently stocked with PPE. Specific items have been added to reduce overall exposure in the ICU cubicles, such as plastic specimen and 24-hour urine containers, buckets with biocide in which instruments are soaked, rubbish-disposal boxes containing two red plastic bags, and X-ray, dialysis and ECG machines and a cardiac output monitor. Linen that has been condemned owing to wear is used and incinerated after use.

Specific infection control procedures have been established, and intensive education, particularly with regard to the donning and doffing of PPE, has been instituted; in the event of an outbreak, 24-hour supervision by infection control staff has been mandated. We believe this to be critical to management of these cases, and it was often not the case in Europe and the USA where transmission occurred. The education and supervision includes the safe performance of invasive procedures and protocols that must be followed when drawing and transporting laboratory specimens.

The stress of managing such cases requires reorganisation of staff schedules; in the CMJAH unit, four nursing staff at a time would work 12-hour shifts, 3 hours on and 3 hours off. A record is kept of all contacts, and all staff are monitored with daily temperature and symptom charts. Baseline platelet count and transaminase values are obtained. Protocols have also been developed for collection and transport of specimens (only the most experienced laboratory staff process samples) and the efficient and safe management of waste. Similarly, procedures have been developed for the management of patients who die.

The World Health Organization and Centers for Disease Control guidelines with regard to PPE have until recently been somewhat contradictory, in that an N95 respirator has been advised for laboratory workers, whereas clinicians have been advised to use surgical masks. We believe this to be illogical, considering that the circumstances in which patients are managed are always more chaotic than those in the controlled laboratory environment, and as such (especially since the viruses are potentially lethal) an N95 mask as a minimum should be mandatory. It should be noted that there remains some uncertainty as to the modes of transmission of viruses such as Ebola, and that many of the procedures involved in management are aerosol generating. ${ }^{[23]}$

\section{Ethics and personal liberties}

An often neglected component of training relates to professional and ethical responsibilities, including responses to unjustified absenteeism and individual liberty and autonomy. While it may be compulsory to provide care for infected patients, HCWs still have to weigh this against competing obligations. ${ }^{[24]}$ In our experience 
there is a remarkable commitment and sense of duty among staff who are involved. We find that regular training in infection control procedures creates a sense of reality that makes staff more likely to consider management of these patients as a routine event. This does not imply negligence, but rather a reduction in the 'fear' element often associated with lapses in infection control. It is critical also that staff designated to care for these possible VHF patients feel supported by the hospital administration and senior staff members, and that they are recognised for the sacrifices that they are called on to make. In the management of multiple patients with a variety of VHFs in various Johannesburg hospitals, no doctor or nurse, or any member of the ancillary staff, has refused to care for the patients. This has been a reflection of the availability of appropriate PPE and the reassurance, leadership and support from clinical heads and infection control staff.

We hope that our experiences in somewhat resource-constrained environments may be of assistance to others faced with similar situations.

1. Gear JS, Cassel GA, Gear AJ, et al. Outbreak of Marburg virus disease in Johannesburg. BMJ 1975;4(5995):489-493.

2. Martini GA. Marburg agent disease in man. Trans R Soc Trop Med Hyg 1969;63(3):295-302.

3. Van Eeden PJ, Joubert JR, van de Wal BW. A nosocomial outbreak of Crimean-Congo haemorrhagic fever at Tygerberg Hospital: Part I. Clinical features. S Afr Med J 1985;68(10):711-717.

fever at Tygerberg Hospital: Part I. Clinical features. S Afr Med J 1985;68(10):711-717.
Centers for Disease Control Crimean-Congo hemorrhagic fever - Republic of South Africa. MMWR Centers for Disease Control Crimean-Congo hem
Morb Mortal Wkly Rep 1985;34(7):94, 99-101.

5. Georges AJ, Leroy EM, Renaud AA, et al. Ebola hemorrhagic fever outbreaks in Gabon, 1994-1997; Epidemiologic and health control issues. J Infect Dis 1999;179(Suppl 1):S65-S75.

6. Richards GA, Murphy S, Jobson R, et al. Unexpected Ebola virus in a tertiary setting: Clinical and epidemiologic aspects. Crit Care Med 2000;28(1):240-224.

. Peters CJ, LeDuc LW. An Introduction to Ebola: The virus and the disease. J Infect Dis 1999;179(Suppl 1):ix-xvi. [http://dx.doi.org/10.1086/514322]

8. Paweska JT, Sewlall NH, Ksiazek TG, et al. Nosocomial outbreak of novel arenavirus infection, southern Africa. Emerg Infect Dis 2009;15(10):1598-1602. [http://dx.doi.org/10.3201/eid1510.090211] 9. Sewlall NH, Richards GA, Duse A, et al. PLoS Negl Trop Dis 2014;8:e3233. [http://dx.doi.org/10.1371/ journal.pntd.0003233
10. Richards GA, Sewlall N, Duse A. Availability of drugs for formidable communicable diseases. Lancet Infect Dis 2009;373(9663):545-546. [http://dx.doi.org/10.1016/S0140-6736(09)60202-6]

11. Risi GF, Bloom ME, Hoe NP, et al. Preparing a community hospital to manage work-related exposures to infectious agents in biosafety level 3 and 4 laboratories. Emerg Infect Dis 2010;16(3):373-378. [http://dx.doi.org/10.3201/eid1603.091485]

12. Kortepeter MG, Martin JW, Rusnak JM, et al. Managing potential laboratory exposure to Ebola virus by using a patient biocontainment care unit. Emerg Infect Dis 2008;14(6):881-887. [http://dx.doi org/10.3201/eid 1406.071489]

13. Randle J, Arthur A, Vaughan N. Twenty-four-hour observational study of hospital hand hygien compliance. J Hosp Infect 2010;76(3):252-255. [http://dx.doi.org/10.1016/j.jhin.2010.06.027]

14. Ryan ET, Wilson ME, Kain KC. Illness after international travel. N Engl J Med 2002;347(7):505-516. [http://dx.doi.org/10.1056/NEJMra020118]

15. O’Brien D, Tobin S, Brown GB, Torresi J. Fever in returned travelers: Review of hospital admissions for a 2-year period. Clin Infect Dis 2001;33(5):603-609. [http://dx.doi.org/10.1086/322602]

16. Doherty JF, Grant $\mathrm{AD}$, Bryceson $\mathrm{AD}$. Fever as the presenting complaint of travellers returning from the tropics. QJM 1995;88(4):277-281.

17. Sprung CL, Zimmerman JL, Christian MD, et al. Recommendations for intensive care unit and hospital preparations for an influenza epidemic or mass disaster: Summary report of the European Society of Intensive Care Medicine's Task Force for intensive care unit triage during an influenza epidemic or Intensive Care Medicine's Task Force for intensive care unit triage during an influenza epidemic o
mass disaster. Intensive Care Med 2010;36(3):428-443. [http://dx.doi.org/10.1007/s00134-010-1759-y]

18. Brouqui P, Puro V, Fusco FM, et al., for the EUNID Working Group. Infection control in the management Bouqui P, Puro V, Fusco FM, et al., for the EUNID Working Group. Infection control in the management
of highly pathogenic infectious diseases: Consensus of the European Network of Infectious Disease. of highly pathogenic infectious diseases: Consensus of the European Network of Infectio
Lancet Infect Dis 2009;9(5):301-311. [http://dx.doi.org/10.1016/S1473-3099(09)70070-2]

19. Wang $\mathrm{C}$, Weia $\mathrm{S}$, Xianga $\mathrm{H}$, et al. Evaluating the effectiveness of an emergency preparedness trainin programme for public health staff in China. Public Health 2008;122(5):471-477. [http://dx.doi org/10.1016/j.puhe.2007.08.006

20. Richards GA, Sprung CL. Educational process: Recommendations and standard operating procedures for intensive care unit and hospital preparations for an influenza epidemic or mass disaster. Intensive Care Med 2010;36(Suppl 1):S70-S79. [http://dx.doi.org/10.1007/s00134-010-1768-x]

21. Lau J, Fung K, Wong T, et al. SARS transmission among hospital workers in Hong Kong. Emerg Infect Dis 2004;10(2):280-286. [http://dx.doi.org/10.3201/eid1002.030534]

22. Rebmann T, English JF, Carrico R. Disaster preparedness lessons learned and future directions for education: Results from focus groups conducted at the 2006 APIC conference. Am J Infect Control 2007;35(6):374-381. [http://dx.doi.org/10.1016/j.ajic.2006.09.002]

23. MacIntyre CR, Chughtai AA, Seale H, Richards GA, Davidson PM. Respiratory protection for healthcare workers treating Ebola virus disease (EVD): Are facemasks sufficient to meet occupational health and safety obligations? Int J Nurs Stud 2014:51(11):1421-1426. [http://dx.doi.org/10.1016/j. ijnurstu.2014.09.002

24. Thompson AK, Faith K, Gibson JL, Upshur REG. Pandemic influenza preparedness: An ethical framework to guide decision-making. BMC Med Ethics 2006;:12 PMID:17144926. [http://dx.doi.org/10.1186/1472-6939-7-12]

Accepted 30 June 2015 